\title{
ON THE EXISTENCE AND BLOW-UP OF SOLUTIONS FOR A MEAN FIELD EQUATION WITH VARIABLE INTENSITIES
}

\author{
T. RICCIARDI*, R. TAKAHASHI, G. ZECCA, AND X. ZHANG
}

\begin{abstract}
We study an elliptic problem with exponential nonlinearities describing the statistical mechanics equilibrium of point vortices with variable intensities. For suitable values of the physical parameters we exclude the existence of blow-up points on the boundary, we prove a mass quantization property and we apply our analysis to the construction of minimax solutions.
\end{abstract}

2010 Mathematics Subject Classification. 35J91, 35B44, 35J20

Key words and phrases. Mean field equation, blow-up solutions, turbulent Euler flow.

\section{IntRoduction AND MAIN RESUlT}

Motivated by the theory of hydrodynamic turbulence as developed by Onsager [8, 17, we consider the problem:

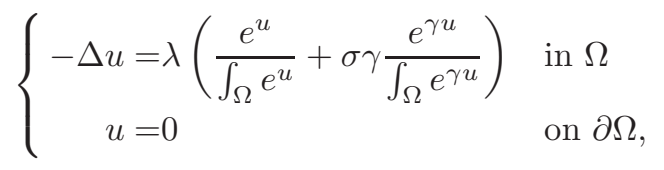

where $\lambda, \sigma>0, \gamma \in[-1,1)$ and $\Omega \subset \mathbb{R}^{2}$ is a smooth bounded domain. Problem (1.1) is derived by statistical mechanics arguments under a "deterministic" assumption on the point vortex intensities [3, 26]. More precisely, the equation derived in 26] is given by

$$
\left\{\begin{aligned}
-\Delta u & =\tilde{\lambda} \int_{[-1,1]} \frac{\alpha e^{\alpha u}}{\int_{\Omega} e^{\alpha u} d x} \mathcal{P}(d \alpha) & & \text { in } \Omega \\
u & =0 & & \text { on } \partial \Omega,
\end{aligned}\right.
$$

where $u$ is the stream function of the two-dimensional flow, $\mathcal{P}$ is a Borel probability measure defined on the interval $[-1,1]$ describing the point vortex intensity distribution and $\widetilde{\lambda}>0$ is a constant related to the inverse temperature. In the special case $\mathcal{P}(d \alpha)=\mathcal{P}_{\gamma}(d \alpha)$, where

$$
\mathcal{P}_{\gamma}(d \alpha)=\tau \delta_{1}(d \alpha)+(1-\tau) \delta_{\gamma}(d \alpha)
$$

and $\delta_{1}(d \alpha), \delta_{\gamma}(d \alpha)$ denote the Dirac measures concentrated at the points $1, \gamma \in[-1,1]$, respectively, and $\tau \in(0,1]$, problem (1.2) takes the form

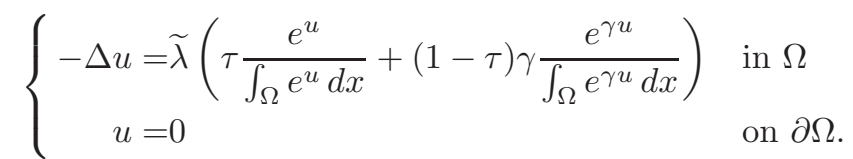

Setting

$$
\lambda=\widetilde{\lambda} \tau, \quad \sigma=\frac{1-\tau}{\tau},
$$

problem (1.4) reduces to (1.1).

Date: September 16, 2015.

* Corresponding author.

This research is partially supported by Programma di scambi internazionali con università ed istituti stranieri per la mobilità breve di docenti, ricercatori e studiosi of Università di Napoli Federico II and by Progetto di ricerca GNAMPA 2015 Alcuni aspetti di equazioni ellittiche non lineari. 
We observe that taking $\gamma=-1$ in problem (1.1) we obtain the sinh-Poisson type problem derived in [19]:

$$
\left\{\begin{array}{cl}
-\Delta u=\lambda\left(\frac{e^{u}}{\int_{\Omega} e^{u}}-\sigma \frac{e^{-u}}{\int_{\Omega} e^{-u}}\right) & \text { in } \Omega \\
u=0 & \text { on } \partial \Omega,
\end{array}\right.
$$

which has received a considerable interest in recent years, see 1, 10, 12, 13, 16, 20 and the references therein. In particular, the blow-up analysis for (1.6) has been clarified by geometrical arguments involving constant mean curvature surfaces in [13]. However, such an approach seems difficult to extend to our case.

For $\sigma=0$ problem (1.1) reduces to the standard mean field problem

$$
\left\{\begin{aligned}
-\Delta u & =\lambda \frac{e^{u}}{\int_{\Omega} e^{u} d x} & & \text { in } \Omega \\
u & =0 & & \text { on } \partial \Omega,
\end{aligned}\right.
$$

which has been extensively analyzed in view of its connections to differential geometry, physics and biology, see, e.g., [11. However, even in the "positive case" $\gamma \in(0,1)$, problem (1.1) does not necessarily exhibit the properties of a perturbation of (1.7). This fact may be seen, for example, by considering the optimal constant for the Moser-Trudinger inequality associated to (1.1), see [21, 28, or the proof of Lemma 3.2 below. In this respect, problem (1.1) significantly differs from its "stochastic" version derived in [14] and recently analyzed in [18, 22, 23, 24, 25]. In fact, our aim in this article is to determine suitable smallness conditions for $|\gamma|$ and $\sigma$ (see (1.8)-(1.9) below) which ensure that the nonlinearity $e^{\gamma u}$ may indeed be treated as "lower-order" with respect to the "principal" term $e^{u}$. In particular, under such smallness conditions we prove the mass quantization for blow-up solution sequences, we derive an improved Moser-Trudinger inequality and we consequently obtain an existence result for solutions in the supercritical range $\lambda>8 \pi$.

More precisely, for every fixed $\gamma$ satisfying $0<|\gamma|<1 / 2$ let

$$
\sigma_{\gamma}:=\frac{1-2|\gamma|}{2 \gamma^{2}}
$$

and

$$
\lambda_{\sigma, \gamma}:=\min \left\{\frac{16 \pi}{1+2 \sigma \gamma^{2}}, \frac{4 \pi}{|\gamma|(1+|\gamma| \sigma)}\right\} .
$$

Our main result is the following.

Theorem 1.1. Assume that $\mathbb{R}^{2} \backslash \Omega$ has a bounded component containing at least one interior point. Fix $0<|\gamma|<1 / 2$ and $0<\sigma<\sigma_{\gamma}$. Then, there exists a solution to Problem (1.1) for every $\lambda \in\left(8 \pi, \lambda_{\sigma, \gamma}\right)$.

We note that $8 \pi<\lambda_{\gamma, \sigma}<16 \pi$ whenever $0<|\gamma|<1 / 2$ and $\sigma \in\left(0, \sigma_{\gamma}\right)$, see Lemma 3.3 below.

Finally, we remark that problem (1.1) shares some similarity in structure with Liouville systems and Toda-type systems. Indeed, setting $v_{1}=G * e^{u}, v_{2}=G * e^{\gamma u}, a^{11}=\lambda / \int_{\Omega} e^{u}$, $a^{12}=\lambda \sigma \gamma / \int_{\Omega} e^{\gamma u}, a^{21}=\gamma \lambda / \int_{\Omega} e^{u}, a^{22}=\gamma^{2} \sigma \lambda / \int_{\Omega} e^{\gamma u}$, we obtain $u=\lambda v_{1} / \int_{\Omega} e^{u} d x+$ $\lambda \sigma \gamma v_{2} / \int_{\Omega} e^{\gamma u}$ and problem (1.1) takes the form $-\Delta v_{i}=\exp \left\{\sum_{j=1,2} a^{i j} v_{j}\right\}, i=1,2$, which is a system of Liouville type, as analyzed in [4, 6]. On the other hand, setting $w_{1}=u$, $w_{2}=\gamma u, b^{11}=\lambda / \int_{\Omega} e^{w_{1}}, b^{12}=\lambda \sigma \gamma / \int_{\Omega} e^{w_{2}}, b^{21}=\lambda \gamma / \int_{\Omega} e^{w_{1}}, b^{22}=\lambda \sigma \gamma^{2} / \int_{e}^{w_{2}}$, we obtain the system $-\Delta w_{i}=\sum_{j=1,2} b^{i j} e^{w_{j}} j=1,2$, which has a "Toda-like" structure when $\gamma<0$, see [1] and the references therein. However, Theorem 1.1] does not follow directly from the results for systems of Liouville and Toda type mentioned above, due to the substantially different assumptions for the coefficients $\left(a^{i j}\right)$ and $\left(b^{i j}\right), i, j=1,2$.

This note is organized as follows. In Section[2 we use Brezis-Merle estimates [2] to exclude the existence of blow-up points on the boundary and to derive a mass quantization property, for suitably small values of $\gamma$ and $\sigma$. We note that the exclusion of boundary blow-up points could also be derived by extending the argument in 22. Here, we provide a simple ad hoc proof which exploits the smallness assumptions on $|\gamma|$ and $\sigma$. In Section 3 we derive an 
improved Moser-Trudinger type inequality. We prove Theorem 1.1 by suitably adapting an argument in [7] and by applying the blow-up results derived in Section 2 ,

Notation. Henceforth, all integrals are taken with respect to the Lebesgue measure. We may omit the integration variables if they are clear from the context. We denote by $C$ a general constant whose actual value may vary from line to line.

\section{BLOW-UP RESUltS}

In this section we show that for suitably small values of $|\gamma|$ and $\sigma$ the blow-up analysis for problem (1.1) is similar to the blow-up analysis for the standard mean field equation (1.7). We first exclude the existence of boundary blow-up points. Then, we prove a mass quantization property.

More precisely, let $\left(u_{n}, \lambda_{n}\right)$ be a solution sequence for (1.1) with $\lambda_{n} \rightarrow \lambda_{0} \geq 0$. We define

$$
\mathcal{S}_{ \pm}=\left\{x_{0} \in \bar{\Omega}: \exists x_{n} \rightarrow x_{0} \text { such that } u_{n}\left(x_{n}\right) \rightarrow \pm \infty\right\}
$$

and we set $\mathcal{S}=\mathcal{S}_{+} \cup \mathcal{S}_{-}$.

2.1. Boundary blow-up exclusion. In the case where $\gamma>0$ the boundary blow-up is readily excluded in view of the moving plane argument in [9], p. 223. Therefore, throughout this subsection, we consider the "asymmetric sinh-case" of (1.1), namely

$$
\left\{\begin{aligned}
-\Delta u & =\lambda\left(\frac{e^{u}}{\int_{\Omega} e^{u}}-\sigma|\gamma| \frac{e^{-|\gamma| u}}{\int_{\Omega} e^{-|\gamma| u}}\right) & & \text { in } \Omega \\
u & =0 & & \text { on } \partial \Omega .
\end{aligned}\right.
$$

We make the following assumption:

$$
\lambda(1+\sigma|\gamma|)<\frac{4 \pi}{|\gamma|}
$$

In this subsection we show the following.

Proposition 2.1. Let $\left(u_{n}, \lambda_{n}\right)$ be a solution sequence for problem (2.1) with $\lambda_{n} \rightarrow \lambda_{0} \geq 0$ and assume that $\lambda_{0}$ satisfies (2.2). Then, $\mathcal{S} \cap \partial \Omega=\emptyset$.

We first reduce problem (2.1) to a mean field type problem with smooth weight function. Let $G=G(x, y)$ be the Green's function defined for $x, y \in \Omega$ by

$$
\left\{\begin{aligned}
-\Delta G(\cdot, y) & =\delta_{y} & & \text { in } \Omega \\
G(\cdot, y) & =0 & & \text { on } \partial \Omega .
\end{aligned}\right.
$$

Let $u:=u_{+}-u_{-}$, where

We observe that

$$
\begin{aligned}
& u_{+}=G * \lambda \frac{e^{u}}{\int_{\Omega} e^{u}} \\
& u_{-}=G * \lambda \sigma|\gamma| \frac{e^{-|\gamma| u}}{\int_{\Omega} e^{-|\gamma| u}} .
\end{aligned}
$$

$$
\left\{\begin{aligned}
-\Delta u_{+} & =\lambda \frac{h(x) e^{u_{+}}}{\int_{\Omega} h(x) e^{u_{+}}} & & \text {in } \Omega \\
u_{+} & =0 & & \text { on } \partial \Omega,
\end{aligned}\right.
$$

where $h(x)=e^{-u_{-}}$satisfies $\|h\|_{C^{1, \alpha}(\bar{\Omega})} \leq C, h \equiv 1$ on $\partial \Omega$. In fact, we have

$$
\left\{\begin{array}{cl}
-\Delta u_{-}=\lambda \sigma|\gamma| \frac{e^{-|\gamma| u}}{\int_{\Omega} e^{-|\gamma| u}} & \text { in } \Omega \\
u_{-}=0 & \text { on } \partial \Omega,
\end{array}\right.
$$

where $\lambda \sigma|\gamma| \frac{e^{-|\gamma| u}}{\int_{\Omega} e^{-|\gamma| u}}$ is $L^{q}$-bounded for some $q>1$. To see this fact, recall from [2] that if $u$ satisfies:

$$
\left\{\begin{aligned}
-\Delta u=f & \text { in } \Omega \\
u=0 & \text { on } \partial \Omega
\end{aligned}\right.
$$


for some $f \in L^{1}(\Omega)$, then for any small $\delta>0$ we have

$$
\int_{\Omega} \exp \left\{\frac{(4 \pi-\delta)}{\|f\|_{L^{1}(\Omega)}}|u|\right\} \leq \frac{4 \pi^{2}}{\delta}(\operatorname{diam} \Omega)^{2} .
$$

Hence, by elliptic estimates, $\left\|u_{-}\right\|_{L^{\infty}(\Omega)} \leq C$. Now we write the equation for $u_{-}$in the form

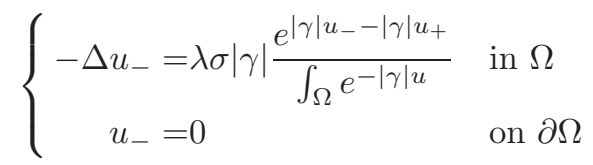

and we observe that since $u_{+} \geq 0$ we have $e^{-|\gamma| u_{+}} \leq 1$. Hence, the right hand side in (2.4) is $L^{\infty}(\Omega)$-bounded. It follows that $\left\|u_{-}\right\|_{W^{2, p}(\Omega)} \leq C$ for every $p \in(1,+\infty)$. In particular, $\left\|u_{-}\right\|_{C^{1, \alpha}(\bar{\Omega})} \leq C$.

Proof of Proposition 2.1. We adapt an argument of 9 p. 223 to our case. Let $x_{0} \in \partial \Omega$ and let $D_{r}$ be a closed disc touching $\bar{\Omega}$ only at $x_{0}$. For convenience we assume $D_{r}=D(0, r)$ and $x_{0}=(r, 0)$. Then, the inversion mapping $x \mapsto y=r^{2} x /|x|^{2}$ fixes the boundary of $D_{r}$ and maps $\Omega$ to a region $y(\bar{\Omega})$ contained inside $D_{r}$. Setting $v(y)=u^{+}(x)$, recalling that

$$
D_{x}=\frac{r^{2}}{|y|^{4}}\left(\begin{array}{cc}
-y_{1}^{2}+y_{2}^{2} & -2 y_{1} y_{2} \\
-2 y_{1} y_{2} & y_{1}^{2}-y_{2}^{2}
\end{array}\right), \quad D_{x}^{T} D_{x}=\frac{r^{4}}{|y|^{4}} I,
$$

where $I$ denotes the identity mapping, we obtain the following equation for $v$ :

$$
\Delta v+\rho \frac{r^{4}}{|y|^{4}} h(x(y)) e^{v}=0 \quad \text { in } y(\bar{\Omega}) .
$$

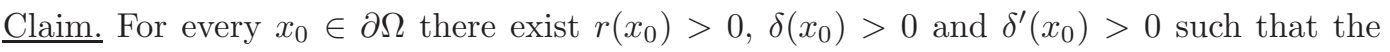
function

$$
H(y)=\frac{r^{4}}{|y|^{4}} h(x(y))
$$

is decreasing in $y_{1}$-direction in the set

$$
\left\{\frac{r\left(x_{0}\right)}{1+\delta\left(x_{0}\right)} \leq|y| \leq r\left(x_{0}\right), y_{1}>0,\left|y_{2}\right| \leq \delta^{\prime}\left(x_{0}\right)\right\} \subset y(\bar{\Omega}),
$$

provided that $r \leq r\left(x_{0}\right)$.

Proof of Claim. In view of (2.5) we compute:

$$
\begin{aligned}
& \partial_{y_{1}} \frac{1}{|y|^{4}}=-\frac{4 y_{1}}{|y|^{6}} \\
& \partial_{y_{1}} h(x(y))=\frac{r^{2}}{|y|^{4}}\left\{\left(\partial_{x_{1}} h(x(y))\right)\left(-y_{1}^{2}+y_{2}^{2}\right)+\left(\partial_{x_{2}} h(x(y))\right)\left(-2 y_{1} y_{2}\right)\right\}
\end{aligned}
$$

and therefore

$$
\begin{aligned}
\frac{1}{r^{4}} \partial_{y_{1}} H(y) & =\frac{1}{|y|^{6}}\left\{-4 y_{1} h(x(y))+\frac{r^{2}}{|y|^{2}}\left[\left(\partial_{x_{1}} h(x(y))\right)\left(-y_{1}^{2}+y_{2}^{2}\right)+\left(\partial_{x_{2}} h(x(y))\right)\left(-2 y_{1} y_{2}\right)\right]\right\} \\
& =\frac{1}{|y|^{6}}\left\{-y_{1}\left[4 h(x(y))+\frac{r^{2}}{|y|^{2}}\left(\partial_{x_{1}} h\right) y_{1}\right]+\frac{r^{2}}{|y|^{2}} y_{2}^{2}\left(\partial_{x_{1}} h\right)+\frac{r^{2}}{|y|^{2}}\left(\partial_{x_{2}} h\right)\left(-2 y_{1} y_{2}\right)\right\} .
\end{aligned}
$$

We estimate, for $|y| \geq r /(1+\delta),\left|y_{2}\right|<\delta^{\prime}$ :

$$
\begin{aligned}
& \left|\frac{r^{2}}{|y|^{2}}\left(\partial_{x_{1}} h\right) y_{1}\right| \leq(1+\delta)^{2}\|h\|_{C^{1}(\bar{\Omega})} r \\
& \left|\frac{r^{2}}{|y|^{2}} y_{2}^{2}\left(\partial_{x_{1}} h\right)\right| \leq(1+\delta)^{2}\|h\|_{C^{1}(\bar{\Omega})}\left(\delta^{\prime}\right)^{2} \\
& \left|\frac{r^{2}}{|y|^{2}}\left(\partial_{x_{2}} h\right)\left(-2 y_{1} y_{2}\right)\right| \leq 2(1+\delta)^{2}\|h\|_{C^{1}(\bar{\Omega})} r \delta^{\prime} .
\end{aligned}
$$

By choosing $r=r\left(x_{0}\right)$ sufficiently small, we achieve

$$
4 h(x(y))+\frac{r^{2}}{|y|^{2}}\left(\partial_{x_{1}} h\right) y_{1} \geq 2 .
$$


Then, for $\delta^{\prime}$ sufficiently small, we have

$$
\begin{aligned}
-y_{1}\left[4 h(x(y))+\frac{r^{2}}{|y|^{2}}\left(\partial_{x_{1}}\right) y_{1}\right] & +\frac{r^{2}}{|y|^{2}} y_{2}^{2}\left(\partial_{x_{1}} h\right)+\frac{r^{2}}{|y|^{2}}\left(\partial_{x_{2}} h\right)\left(-2 y_{1} y_{2}\right) \\
\leq & -2 y_{1}+(1+\delta)^{2}\|h\|_{C^{1}(\bar{\Omega})}\left(\delta^{\prime}\right)^{2}+2(1+\delta)^{2}\|h\|_{C^{1}(\bar{\Omega})} r \delta^{\prime} \\
\leq & -\frac{r}{2}<0 .
\end{aligned}
$$

Now the argument in [9] concludes the proof.

2.2. Mass quantization. In view of Proposition 2.1 we have $\mathcal{S} \cap \partial \Omega=\emptyset$. Therefore, by local blow-up results from [15, 23, we know that setting

$$
\mu_{1}(d x):=\lambda \frac{e^{u}}{\int_{\Omega} e^{u}} d x, \quad \quad \mu_{\gamma}(d x):=\lambda \frac{e^{\gamma u}}{\int_{\Omega} e^{\gamma u}} d x
$$

we have

$$
\begin{aligned}
& \mu_{1}(d x) \stackrel{*}{\rightarrow} \sum_{p \in \mathcal{S}} m_{1}(p) \delta_{p}(d x)+r_{1}(x) d x \\
& \mu_{\gamma}(d x) \stackrel{*}{\rightarrow} \sum_{p \in \mathcal{S}} m_{\gamma}(p) \delta_{p}(d x)+r_{\gamma}(x) d x .
\end{aligned}
$$

Lemma 2.1. At every fixed $p \in \mathcal{S}$ we have the quadratic identity:

$$
8 \pi\left(m_{1}(p)+\sigma m_{\gamma}(p)\right)=\left(m_{1}(p)+\sigma \gamma m_{\gamma}(p)\right)^{2} .
$$

Proof. We recall from [15] that if $\left(u_{k}, \widetilde{\lambda}_{k}\right)$ is a solution sequence for (1.4) with

$$
\widetilde{\lambda} \frac{e^{u_{k}}}{\int_{\Omega} e^{u_{k}}} \stackrel{*}{*} \sum_{p \in \mathcal{S}} \widetilde{m}_{1}(p) \delta_{p}(d x)+\widetilde{r}_{1}(x), \quad \widetilde{\lambda} \frac{e^{\gamma u_{k}}}{\int_{\Omega} e^{\gamma u_{k}}} \stackrel{*}{*} \sum_{p \in \mathcal{S}} \widetilde{m}_{\gamma}(p) \delta_{p}(d x)+\widetilde{r}_{\gamma}(x),
$$

where $\delta_{p}(d x)$ denotes the Dirac mass centered at $p \in \Omega$, then the following relation holds:

$$
8 \pi\left(\tau \widetilde{m}_{1}(p)+(1-\tau) \widetilde{m}_{\gamma}(p)\right)=\left(\tau \widetilde{m}_{1}(p)+(1-\tau) \gamma \widetilde{m}_{\gamma}(p)\right)^{2},
$$

for every $p \in \mathcal{S}$. In view of (1.5) we have $\tau \widetilde{m}_{1}(p)=m_{1}(p)$ and $(1-\tau) \widetilde{m}_{\gamma}(p)=\sigma m_{\gamma}(p)$. Hence, we derive the asserted identity. Alternatively, we may derive identity (2.7) by applying the Pohozaev identity in a standard way.

Lemma 2.2. Let $u_{n}$ be a solution sequence for (1.1). For any $\gamma \in[-1,1]$ we have

$$
\int_{\Omega} e^{\gamma u_{n}} \geq c_{0}>0
$$

Proof. If $\gamma>0$, we have $u_{n} \geq 0$ in $\Omega$ by the maximum principle and therefore

$$
\int_{\Omega} e^{\gamma u_{n}} \geq|\Omega|>0
$$

Therefore, we assume $\gamma<0$. We note that since $\left\|u_{n}\right\|_{W_{0}^{1, q}(\Omega)} \leq C$ for any $q \in[1,2)$, there exists $u_{0} \in W_{0}^{1, q}(\Omega)$ such that $u_{n} \rightarrow u_{0}$ weakly in $W_{0}^{1, q}(\Omega)$, strongly in $L^{p}(\Omega)$ for any $p \geq 1$ and a.e. in $\Omega$. In view of Fatou's lemma, we derive

$$
\liminf _{n \rightarrow \infty} \int_{\Omega} e^{\gamma u_{n}} \geq \int_{\Omega} e^{\gamma u_{0}}>0
$$

Proposition 2.2 (Mass quantization). Let $\left(u_{n}, \lambda_{n}\right)$ be a solution sequence for (1.1) with $\lambda_{n} \rightarrow \lambda_{0}$. Assume that $|\gamma|<1 / 2$ and $\sigma \in\left(0, \sigma_{\gamma}\right)$, where $\sigma_{\gamma}$ is defined in (1.8). Moreover, assume that

$$
8 \pi<\lambda_{0}<\frac{4 \pi}{|\gamma|(1+|\gamma| \sigma)} .
$$

Then, we have $m_{\gamma}(p)=0$ and consequently $m_{1}(p)=8 \pi, r_{1} \equiv 0$ and $\lambda_{0} \in 8 \pi \mathbb{N}$. 
Proof. Throughout this proof we omit the subscript $n$. Similarly as above, in view of (2.3) with $f=\lambda\left(\frac{e^{u}}{\int_{\Omega} e^{u}}+\sigma \gamma \frac{e^{\gamma u}}{\int_{\Omega} e^{\gamma u}}\right),\|f\|_{L^{1}(\Omega)} \leq \lambda(1+\sigma|\gamma|)$, we have that $\left\|e^{\gamma u}\right\|_{L^{q}(\Omega)}$ is bounded if $1<q<4 \pi /[\lambda|\gamma|(1+\sigma|\gamma|)]$. The existence of such a $q>1$ follows from (2.8). Moreover, since by assumption we have $\lambda>8 \pi$, we derive that necessarily

$$
8 \pi<\frac{4 \pi}{|\gamma|(1+\sigma|\gamma|)} .
$$

This inequality holds in view of the assumption $\sigma \in\left(0, \sigma_{\gamma}\right)$. Therefore we have that $1<$ $4 \pi /[\lambda|\gamma|(1+\sigma|\gamma|)]$ and $\left\|e^{\gamma u}\right\|_{L^{q}(\Omega)}$ is bounded for some $q>1$. Hence, $m_{\gamma}(p)=0$. Now (2.7) implies $m_{1}(p)=8 \pi$. We decompose $u=w_{1}+w_{2}$, with

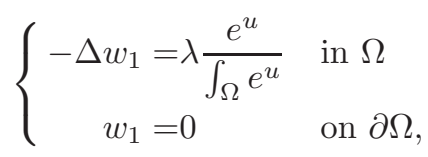

and

Then, setting

$$
\left\{\begin{array}{clrl}
-\Delta w_{2} & =\sigma \gamma \lambda \frac{e^{\gamma u}}{\int_{\Omega} e^{\gamma u}} & & \text { in } \Omega \\
w_{2}=0 & & \text { on } \partial \Omega
\end{array}\right.
$$

$$
\phi=\sigma \gamma \lambda \frac{e^{\gamma u}}{\int_{\Omega} e^{\gamma u}}
$$

we have $\|\phi\|_{L^{q}(\Omega)} \leq C$ for some $q>1$ and therefore $\left\|w_{2}\right\|_{L^{\infty}(\Omega)} \leq C$. It follows that $e^{u}=h e^{w_{1}}$ with $h=e^{w_{2}} \geq e^{\inf _{\Omega} w_{2}} \geq e^{-C(\lambda)}>0$. Moreover,

$$
w_{1} \rightarrow G *\left(m_{1}(p) \delta_{p}+r_{1}\right)=4 \log \frac{1}{|x-p|}+\omega+G * r_{1}
$$

with $\omega$ smooth in the closure of a neighbourhood $U$ of $p$. Therefore, by Fatou's lemma:

$$
\liminf \int_{\Omega} e^{u} \geq \int_{\Omega} \liminf e^{u} \geq e^{-C(\lambda)} \int_{U} e^{\omega} \frac{d x}{|x-p|^{4}}=+\infty .
$$

Hence $r_{1} \equiv 0$ since $u$ is locally uniformly bounded in $\Omega \backslash \mathcal{S}$.

Now, the first equation in (2.6) implies the mass quantization $\lambda \in 8 \pi \mathbb{N}$.

\section{Proof of Theorem 1.1}

In this section we prove Theorem 1.1 by suitably adapting a variational argument due to [7. The variational functional for Problem (1.1) is given by:

$$
J_{\lambda}(u)=\frac{1}{2} \int_{\Omega}|\nabla u|^{2} d x-\lambda \ln \int_{\Omega} e^{u} d x-\lambda \sigma \ln \int_{\Omega} e^{\gamma u} d x .
$$

3.1. An improved Moser-Trudinger inequality. We observe that the standard wellknown improved Moser-Trudinger inequality [5] readily implies an improved inequality for $J_{\lambda}$. For any fixed $a_{0}, d_{0}>0$ we consider the set

$\mathcal{A}_{a_{0}, d_{0}}:=\left\{u \in H_{0}^{1}(\Omega): \exists \Omega_{1}, \Omega_{2} \subset \Omega\right.$ s.t. $\operatorname{dist}\left(\Omega_{1}, \Omega_{2}\right) \geq d_{0}$ and $\left.\frac{\int_{\Omega_{i}} e^{u} d x}{\int_{\Omega} e^{u} d x} \geq a_{0}, i=1,2\right\}$.

Lemma 3.1 (Improved Moser-Trudinger Inequality). The functional $J_{\lambda}$ is bounded from below on $\mathcal{A}_{a_{0}, d_{0}}$ if

$$
\lambda<\frac{16 \pi}{1+2 \sigma \gamma^{2}} .
$$

Proof. For $\epsilon \in(0,1)$ to be fixed later we decompose:

$$
\begin{aligned}
J_{\lambda}(u)=(1-\epsilon) & \left\{\frac{1}{2} \int_{\Omega}|\nabla u|^{2} d x-\frac{\lambda}{1-\epsilon} \ln \int_{\Omega} e^{u}\right\} \\
+ & \frac{\epsilon}{\gamma^{2}}\left\{\frac{1}{2} \int_{\Omega}|\nabla \gamma u|^{2} d x-\frac{\lambda \sigma \gamma^{2}}{\epsilon} \ln \int_{\Omega} e^{\gamma u}\right\} \\
:=K^{1}(u) & +K^{\gamma}(u)
\end{aligned}
$$


In view of the Improved Moser-Trudinger inequality, the functional $K^{1}$ is bounded below on $\mathcal{A}_{a_{0}, d_{0}}$ if

$$
\frac{\lambda}{1-\epsilon}<16 \pi .
$$

On the other hand, the functional $K^{\gamma}$ is bounded below on $H_{0}^{1}(\Omega)$ if

$$
\frac{\lambda \sigma \gamma^{2}}{\epsilon} \leq 8 \pi
$$

Considering (3.3) and (3.4) we can take a suitable $\epsilon \in(0,1)$ satisfying

if

$$
\epsilon<1-\frac{\lambda}{16 \pi} \quad \text { and } \quad \epsilon \geq \frac{\lambda \sigma \gamma^{2}}{8 \pi}
$$

which is equivalent to (3.1).

$$
\frac{\lambda \sigma \gamma^{2}}{8 \pi}<1-\frac{\lambda}{16 \pi}
$$

Remark 3.1. Actually, we expect boundedness below of $J_{\lambda}$ for all $\lambda \in(8 \pi, 16 \pi)$.

Lemma 3.2. For every $0<|\gamma|<1 / 2$ and for every $0<\sigma<(1-2|\gamma|) /\left(2 \gamma^{2}\right)=\sigma_{\gamma}$, the functional $J_{\lambda}$ is bounded below on $H_{0}^{1}(\Omega)$ if and only if $\lambda \leq 8 \pi$.

Proof. We rewrite

$$
J_{\lambda}(u)=\tilde{J}_{\tilde{\lambda}}(u)=\frac{1}{2} \int_{\Omega}|\nabla u|^{2} d x-\tilde{\lambda} \tau \ln \int_{\Omega} e^{u} d x-\tilde{\lambda}(1-\tau) \ln \int_{\Omega} e^{\gamma u} d x .
$$

where:

$$
\sigma=\frac{1-\tau}{\tau} \quad \text { and } \quad \tilde{\lambda}=\frac{\lambda}{\tau} \quad \tau \in(0,1] .
$$

We use a result from 21] for the functionals of the form

$$
J_{\tilde{\lambda}}^{\mathcal{P}}(u)=\frac{1}{2} \int_{\Omega}|\nabla u|^{2} d x-\tilde{\lambda} \int_{I}\left(\log \int_{\Omega} e^{\alpha u} d x\right) \mathcal{P}(d \alpha),
$$

$u \in H_{0}^{1}(\Omega)$. Note that $J_{\tilde{\lambda}}^{\mathcal{P}}$ is the Euler-Lagrange funtional for problem (1.2). In view of Theorem 4 in 21] (see also [27]) we have that $\tilde{J}_{\tilde{\lambda}}$ is bounded below if and only if $\tilde{\lambda} \leq \bar{\lambda}^{\mathcal{P}}$ where

$$
\bar{\lambda}^{\mathcal{P}}=8 \pi \inf \left\{\frac{\mathcal{P}\left(K_{ \pm}\right)}{\left(\int_{K_{ \pm}} \alpha \mathcal{P}(d \alpha)\right)^{2}}, K_{ \pm} \subset I_{ \pm} \cap \operatorname{supp} \mathcal{P}\right\}
$$

$I_{+}=[0,1], I_{-}=[-1,0)$ and $\mathcal{P}=\mathcal{P}_{\gamma}$ is defined by (1.3), i.e., $\mathcal{P}_{\gamma}(d \alpha)=\tau \delta_{1}(d \alpha)+(1-$ $\tau) \delta_{\gamma}(d \alpha)$.

Assume $\gamma \geqslant 0$. In this case, we have

$$
\tau \frac{\mathcal{P}_{\gamma}(K)}{\left(\int_{K} \alpha \mathcal{P}_{\gamma}(d \alpha)\right)^{2}}= \begin{cases}1, & \text { if } K=\{1\} \\ \frac{\tau}{\gamma^{2}(1-\tau)}=\frac{1}{\sigma \gamma^{2}}, & \text { if } K=\{\gamma\} \\ \frac{\tau}{(\tau+\gamma(1-\tau))^{2}}=\frac{1+\sigma}{(1+\sigma \gamma)^{2}}, & \text { if } K=\{\gamma, 1\} .\end{cases}
$$

Hence, if $\gamma>0$ we have $\tau \bar{\lambda}^{\mathcal{P}}=8 \pi$ whenever $0<\sigma \leqslant \frac{1-2 \gamma}{2 \gamma^{2}}=\sigma_{\gamma}$.

Analogously, for $\gamma<0$ we have

$$
\tau \frac{\mathcal{P}_{\gamma}(K)}{\left(\int_{K} \alpha \mathcal{P}_{\gamma}(d \alpha)\right)^{2}}= \begin{cases}1, & \text { if } K=\{1\} \\ \frac{\tau}{\gamma^{2}(1-\tau)}=\frac{1}{\sigma \gamma^{2}}, & \text { if } K=\{\gamma\} .\end{cases}
$$

Hence, if $\gamma<0$ we have that $\tau \bar{\lambda}^{\mathcal{P}}=8 \pi$ if $0<\sigma<\sigma_{\gamma}$.

Lemma 3.3. Let $0<|\gamma|<1 / 2$ and let $0<\sigma<\sigma_{\gamma}$, where $\sigma_{\gamma}$ is defined in (1.8). Then, we have $8 \pi<\lambda_{\gamma, \sigma} \leq 16 \pi$, where $\lambda_{\gamma, \sigma}$ is defined in (1.9). 
Proof. The upper bound is clear. Therefore, we only prove the lower bound $\lambda_{\gamma, \sigma}>8 \pi$. We readily check that

$$
\frac{16 \pi}{1+2 \sigma \gamma^{2}}>8 \pi \quad \text { if and only if } \quad \sigma<\frac{1}{2 \gamma^{2}}
$$

and

$$
\frac{4 \pi}{|\gamma|(1+\sigma|\gamma|)}>8 \pi \quad \text { if and only if } \quad \sigma<\sigma_{\gamma}=\frac{1-2|\gamma|}{2 \gamma^{2}}<\frac{1}{2 \gamma^{2}} .
$$

The claim follows.

For every $u \in H_{0}^{1}(\Omega)$ we consider the measure:

$$
\mu_{u}=\frac{e^{u}}{\int_{\Omega} e^{u} d x} d x \in \mathcal{M}(\Omega)
$$

and the corresponding "center of mass":

$$
\bar{x}_{\mu}(u)=\int_{\Omega} x d \mu_{u} \in \mathbb{R}^{2} .
$$

Lemma 3.4. Let $\lambda \in\left(8 \pi, \frac{16 \pi}{1+2 \sigma \gamma^{2}}\right)$ and let $\left\{u_{n}\right\} \subset H_{0}^{1}(\Omega)$ be a sequence such that $J_{\lambda}\left(u_{n}\right) \rightarrow$ $-\infty$ and $\bar{x}_{\mu}\left(u_{n}\right) \rightarrow x_{0} \in \mathbb{R}^{2}$. Then, $x_{0} \in \bar{\Omega}$ and

$$
\mu_{u_{n}} \rightarrow \delta_{x_{0}} \quad \text { weakly*in } \mathcal{C}(\bar{\Omega})^{\prime} .
$$

Proof. For every fixed $r>0$ we denote by $\mathcal{Q}_{n}(r)$ the concentration function of $\mu_{n}$, i.e.

$$
\mathcal{Q}_{n}(r)=\sup _{x \in \Omega} \int_{B(x, r) \cap \Omega} \mu_{n} .
$$

For every $n$, there exists $\tilde{x}_{n} \in \bar{\Omega}$ such that

$$
\mathcal{Q}_{n}(r / 2)=\int_{B\left(\tilde{x}_{n}, r / 2\right) \cap \Omega} \mu_{n} .
$$

Upon taking a subsequence, we have that $\tilde{x}_{n} \rightarrow \tilde{x}_{0} \in \bar{\Omega}$.

Now, let us set

$$
\Omega_{1}^{n}=B\left(\tilde{x}_{n}, r / 2\right) \cap \Omega \quad \text { and } \quad \Omega_{2}^{n}=\Omega \backslash B\left(\tilde{x}_{n}, r\right),
$$

so that

$$
\operatorname{dist}\left(\Omega_{1}^{n}, \Omega_{2}^{n}\right) \geqslant r / 2 .
$$

Since $J_{\lambda}\left(u_{n}\right) \rightarrow-\infty$ and since $\lambda<\frac{16 \pi}{1+2 \sigma \gamma^{2}}$, in view of (3.2) necessarily we have $K^{1}\left(u_{n}\right) \rightarrow$ $-\infty$. Therefore, in view of the standard Improved Moser-Trudinger inequality [5], we conclude that

$$
\min \left\{\mu_{n}\left(\Omega_{1}^{n}\right), \mu_{n}\left(\Omega_{2}^{n}\right)\right\} \rightarrow 0 .
$$

In particular, $\min \left\{\mathcal{Q}_{n}(r / 2), 1-\mathcal{Q}_{n}(r)\right\} \leqslant \min \left(\mu_{n}\left(\Omega_{1}^{n}\right), \mu_{n}\left(\Omega_{2}^{n}\right)\right) \rightarrow 0$.

On the other hand, for every fixed $r>0$ let $k_{r} \in \mathbb{N}$ be such that $\Omega$ is covered by $k_{r}$ balls of radius $r / 2$. Then, $1=\mu_{n}(\Omega) \leqslant k_{r} Q_{n}(r / 2)$, so that $Q_{n}(r / 2) \geqslant k_{r}^{-1}$ for every $n$. We conclude that necessarily $\mathcal{Q}_{n}(r) \rightarrow 1$ as $n \rightarrow \infty$. Since $r>0$ is arbitrary, we derive in turn that $1-\mathcal{Q}_{n}(r / 2)=\mu_{n}\left(\Omega \backslash B\left(\tilde{x}_{n}, r / 2\right)\right) \rightarrow 0$ as $n \rightarrow \infty$. That is, $\mu_{u_{n}} \rightarrow \delta_{\tilde{x}_{0}}$. It follows that $\bar{x}_{\mu}\left(u_{n}\right)=\int_{\Omega} x d \mu_{n} \rightarrow \tilde{x}_{0}=x_{0} \in \bar{\Omega}$, as asserted.

At this point, in order to prove Theorem 1.1, we shall adapt a construction in 7. Let $\Gamma_{1} \subset \Omega$ be a non-contractible curve which exists since $\Omega$ is non-simply connected. Let $\mathbb{D}=\{(r, \theta): 0 \leqslant r<1,0 \leqslant \theta<2 \pi\}$ be the unit disc. Define

$\mathcal{D}_{\lambda}:=\left\{h \in C\left(\mathbb{D}, H_{0}^{1}(\Omega)\right)\right.$ s.t.:

$$
\left.\begin{array}{l}
\lim _{r \rightarrow 1} \sup _{\theta \in[0,2 \pi)} J_{\lambda}(h(r, \theta))=-\infty \\
\bar{x}_{\mu}(h(r, \theta)) \text { can be extended continuously to } \overline{\mathbb{D}} \\
\bar{x}_{\mu}(h(1, \cdot)) \text { is one-to-one from } \partial \mathbb{D} \text { onto } \Gamma_{1}
\end{array}\right\}
$$

Lemma 3.5. For every $\lambda \in(8 \pi, 16 \pi)$ the set $\mathcal{D}_{\lambda}$ is non-empty. 
Proof. Let $\gamma_{1}(\theta):[0,2 \pi) \rightarrow \Gamma_{1}$ be a parametrization of $\Gamma_{1}$ and let $\varepsilon_{0}>0$ be sufficiently small so that $B\left(\gamma_{1}(\theta), \varepsilon_{0}\right) \subset \Omega$. Let $\varphi_{\theta}(x)=\varepsilon_{0}^{-1}\left(x-\gamma_{1}(\theta)\right)$ so that $\varphi_{\theta}\left(B\left(\gamma_{1}(\theta), \varepsilon_{0}\right)\right)=B(0,1)$. We define "truncated Green's function":

$$
V_{r}(X)= \begin{cases}4 \log \frac{1}{1-r} & \text { for } X \in B(0,1-r) \\ 4 \log \frac{1}{|X|} & \text { for } X \in B(0,1) \backslash B(0,1-r)\end{cases}
$$

and

$$
v_{r, \theta}(x)=\left\{\begin{array}{l}
\left.0 \quad \text { for } x \in \Omega \backslash B\left(\gamma_{1}(\theta), \varepsilon_{0}\right)\right) \\
V_{r}\left(\varphi_{\theta}(x)\right) \quad \text { for } x \in B\left(\gamma_{1}(\theta), \varepsilon_{0}\right) .
\end{array}\right.
$$

We set

$$
h(r, \theta)(x)=v_{r, \theta}(x), x \in \Omega .
$$

The function $h$ defined in (3.6) satisfies $h \in \mathcal{D}_{\lambda}$. To see that $h$ verifies the $(i)$-condition it is sufficient to note that

$$
\int_{\Omega} e^{\gamma h} d x \geqslant|\Omega|-\pi \varepsilon_{0}>0
$$

Then the claim follows by [7.

Define

$$
c_{\lambda}:=\inf _{h \in \mathcal{D}_{\lambda}} \sup _{(r, \theta) \in \mathbb{D}} J_{\lambda}(h(r, \theta)) .
$$

We shall prove that $c_{\lambda}$ defines a critical value for $J_{\lambda}$ using the Struwe Monotonicity Trick contained in 25], Proposition 4.1.

In view of Lemma 3.5, we have $c_{\lambda}<+\infty$.

Lemma 3.6. For any $\lambda \in\left(8 \pi, \lambda_{\gamma, \sigma}\right), c_{\lambda}>-\infty$.

Proof. Denote by $B$ a bounded component of $\mathbb{R}^{2} \backslash \Omega$ with at least an interior point and such that $\Gamma_{1}$ encloses $B$. By continuity and by the (iii)-property defining $\mathcal{D}_{\lambda}$, we have $\bar{x}_{\mu}(h(\mathbb{D})) \supset B$ for all $h \in \mathcal{D}_{\lambda}$. By contradiction, assume that $c_{\lambda}=-\infty$. Then, there exists a sequence $\left\{h_{n}\right\} \subset \mathcal{D}_{\lambda}$ such that $\sup _{(r, \theta) \in \mathbb{D}} J_{\lambda}\left(h_{n}(r, \theta)\right) \rightarrow-\infty$. Let $x_{0}$ be an interior point of $B$. For every $n$ we take $\left(r_{n}, \theta_{n}\right) \in \mathbb{D}$ such that $\bar{x}_{\mu}\left(h_{n}\left(r_{n}, \theta_{n}\right)\right)=x_{0}$. In view of Lemma 3.4 it should be $x_{0} \in \stackrel{o}{B} \cap \bar{\Omega}=\emptyset$, a contradiction.

At this point we set

$$
\mathcal{G}(u)=\ln \int_{\Omega} e^{u} d x+\sigma \ln \int_{\Omega} e^{\gamma u} d x
$$

so that our functional (3.5) takes the form

$$
J_{\lambda}(u)=\frac{1}{2} \int_{\Omega}|\nabla u|^{2}-\lambda \mathcal{G}(u) .
$$

Lemma 3.7. For $8 \pi<\lambda_{1} \leqslant \lambda_{2}<16 \pi$, we have $\mathcal{D}_{\lambda_{1}} \subseteq \mathcal{D}_{\lambda_{2}}$.

Proof. It is sufficient to note that whenever $J_{\lambda}(u) \leqslant 0$ it is $\mathcal{G}(u) \geqslant 0$, with $\mathcal{G}$ given by (3.8) and this implies that

$$
J_{\lambda_{1}}(u) \geqslant J_{\lambda_{2}}(u) \quad \text { for } \quad 8 \pi<\lambda_{1} \leqslant \lambda_{2}<16 \pi \quad \text { if } J_{\lambda_{1}}(u) \leqslant 0 .
$$

Hence, $\mathcal{D}_{\lambda_{1}} \subseteq \mathcal{D}_{\lambda_{2}}$ for every $8 \pi<\lambda_{1} \leqslant \lambda_{2}<16 \pi$.

Lemma 3.8. The function $\mathcal{G}: H_{0}^{1}(\Omega) \rightarrow \mathbb{R}$ defined by (3.8) satisfies:

1) $\mathcal{G} \in \mathcal{C}^{2}\left(H_{0}^{1}(\Omega) ; \mathbb{R}\right)$

2) $\mathcal{G}^{\prime}$ is compact

3) $\left\langle\mathcal{G}^{\prime \prime}(u) \varphi, \varphi\right\rangle \geqslant 0$ for every $u, \varphi \in H_{0}^{1}(\Omega)$, where $\langle\cdot, \cdot\rangle$ is the $L^{2}$-inner product. 
Proof. For every $u, \varphi \in H_{0}^{1}(\Omega)$ we have:

$$
\mathcal{G}^{\prime}(u) \varphi=\frac{\int_{\Omega} \varphi e^{u} d x}{\int_{\Omega} e^{u} d x}+\sigma \frac{\int_{\Omega} \gamma \varphi e^{\gamma u} d x}{\int_{\Omega} e^{\gamma u} d x}
$$

and therefore the compactness of $\mathcal{G}^{\prime}$ follows by the compactness of the Moser-Trudinger embedding. Moreover, for every $u, \varphi \in H_{0}^{1}(\Omega)$ we have, using the Schwarz inequality,

$$
\begin{aligned}
\left\langle\mathcal{G}^{\prime \prime}(u) \varphi, \varphi\right\rangle & =\frac{1}{\left(\int_{\Omega} e^{u} d x\right)^{2}}\left[\left(\int_{\Omega} e^{u} \varphi^{2} d x\right)\left(\int_{\Omega} e^{u} d x\right)-\left(\int_{\Omega} e^{u} \varphi d x\right)^{2}\right] \\
& +\frac{\gamma^{2} \sigma}{\left(\int_{\Omega} e^{\gamma u} d x\right)^{2}}\left[\left(\int_{\Omega} e^{\gamma u} \varphi^{2} d x\right)\left(\int_{\Omega} e^{\gamma u} d x\right)-\left(\int_{\Omega} e^{\gamma u} \varphi d x\right)^{2}\right] \geqslant 0 .
\end{aligned}
$$

Now we are able to prove the following.

Proposition 3.1. Let $\sigma>0$ and assume that (1.8) holds. For almost every $\lambda \in\left(8 \pi, \lambda_{\gamma, \sigma}\right)$, $c_{\lambda}>-\infty$ given by (3.7) is a saddle-type critical value for $J_{\lambda}$.

Proof of Proposition 3.1. In view of Lemma 3.8, Lemma 3.5 Lemma 3.6 and Lemma 3.7 we may apply the well known Struwe's monotonicity trick to derive the existence of the desired critical value. See [7] or [25, Proposition 4.1 with $\mathcal{H}=H_{0}^{1}(\Omega), V=\mathbb{D}, A=-\infty$ and $\mathcal{F}_{\lambda}=\mathcal{D}_{\lambda}$.

Proof of Theorem 1.1. (Completion by blow-up results). We fix $\lambda_{0} \in\left(8 \pi, \lambda_{\gamma, \sigma}\right)$. In view of Proposition 3.1 there exists $\lambda_{n} \rightarrow \lambda_{0}$ such that problem (1.1) with $\lambda=\lambda_{n}$ admits a solution $u_{n}$. By the blow up analysis as stated in Proposition 2.2, we have the compactness of solution sequences. Therefore, up to subsequences, we obtain that $u_{n} \rightarrow u_{0}$ with $u_{0}$ a solution to (1.1) with $\lambda=\lambda_{0}$.

\section{REFERENCES}

[1] Battaglia, L., Jevnikar, A., Malchiodi, A., Ruiz, D., A general existence result for Toda system on compact surfaces, arXiv:1306.5404 4 .

[2] Brezis, H., Merle, F., Uniform estimates and blow-up behavior for solutions of $-\Delta u=V(x) e^{u}$ in dimension 2, Comm. Partial Differential Equations 16 (1991), 1223-1253.

[3] Caglioti, E., Lions, P.L., Marchioro, C., Pulvirenti, M.: A special class of stationary flows for twodimensional Euler equations: a statistical mechanics description. Comm. Math. Phys. 143 (1992), $501-525$.

[4] Chanillo, S., Kiessling, M.K.-H., Conformally invariant systems of nonlinear PDE of Liouville type, Geometric And Functional Analysis 5 no. 6 (1995), 924-947.

[5] Chen, W., Li, C., Prescribing Gaussian Curvatures on Surfaces with Conical Singularities, J. of Geometric Analysis 1 n. 4 (1991), 359-372.

[6] Chipot, M., Shafrir, I., Wolansky, G., On the Solutions of Liouville Systems, J. Differential Equations 140 (1997), 59-105.

[7] Ding, W., Jost, J., Li, J., Wang, G., Existence results for mean field equations, Ann. Inst. H. Poincaré, 16 n. 5 (1999), 653-666.

[8] Eyink, G.L., Sreenivasan, K.R., Onsager and the theory of hydrodynamic turbulence, Reviews of Modern Physics 78 (2006), 87-135.

[9] Gidas, B., Ni, W., Nirenberg, L., Symmetry and Related Properties via the Maximum Principle, Commun. Math. Phys. 68, 209-243 (1979).

[10] Grossi, M., Pistoia, A., Multiple Blow-Up Phenomena for the Sinh-Poisson Equation, Arch. Rational Mech. Anal. 209 (2013) 287-320.

[11] Lin, C.S., An expository survey on recent development of mean field equations, Discr. Cont. Dynamical Systems 19 n. 2 (2007), 217-247.

[12] Jevnikar, A., An existence result for the mean field equation on compact surfaces in a doubly supercritical regime, Proc. Roy. Soc. Edinburgh Sect A 143, no. 5 (2013), 1021-1045.

[13] Jost, J., Wang, G., Ye, D., Zhou, C., The blow up of solutions of the elliptic sinh-Gordon equation, Calc. Var. Partial Differential Equations 31 (2008) no. 2, 263-276.

[14] Neri, C., Statistical Mechanics of the $N$-point vortex system with random intesities on a bounded domain, Ann. I. H. Poincaré - AN 21 (2004), 381-399.

[15] Ohtsuka, H., Ricciardi, T., Suzuki, T., Blow-up analysis for an elliptic equation describing stationary vortex flows with variable intensities in 2D-turbulence, J. Differential Equations 249 n. 6 (2010), 14361465. 
[16] Ohtsuka, H., Suzuki, T.: Mean field equation for the equilibrium turbulence and a related functional inequality. Adv. Differential Equations 11, 281-304 (2006).

[17] Onsager, L., Statistical hydrodynamics. Nuovo Cimento Suppl. n. 26 (9), 279-287 (1949).

[18] Pistoia, A., Ricciardi, T.: Concentrating solutions for a Liouville type equation with variable intensities in 2D-turbulence, arXiv:1505.05304

[19] Pointin, Y.B., Lundgren, T.S., Statistical mechanics of two-dimensional vortices in a bounded container, Physics of Fluids 19 no. 10 (1976), 1459-1470.

[20] Ricciardi, T., Mountain pass solutions for a mean field equation from two-dimensional turbulence, Diff. Int. Eqs. 20 no. 5 (2007), 561-575.

[21] Ricciardi, T., Suzuki, T., Duality and best constant for a Trudinger-Moser inequality involving probability measures, J. of Eur. Math. Soc. (JEMS) 16 (2014), 1327-1348.

[22] Ricciardi, T., Takahashi, R., Blow-up behavior for a degenerate elliptic sinh-Poisson equation with variable intensities, arXiv:1507.01449

[23] Ricciardi, T., Zecca, G., Blow-up analysis for some mean field equations involving probability measures from statistical hydrodynamics, Differential and Integral Equations 25 n. 3-4 (2012), 201-222.

[24] Ricciardi, T., Zecca, G., On the blow-up of solutions to Liouville type equations, to appear on Advanced Nonlinear Studies.

[25] Ricciardi, T., Zecca, G., Mass quantization and minimax solutions for Neri's mean field equation in 2D-turbulence, J. Differential Equations (2015) http://dx.doi.org/10.1016/j.jde.2015.08.045

[26] Sawada, K., Suzuki, T., Derivation of the equilibrium mean field equations of point vortex and vortex filament system, Theoret. Appl. Mech. Japan 56 (2008), 285-290.

[27] Shafrir, I., Wolansky, G., The logarithmic HLS inequality for systems on compact manifolds, J. Funct. Anal. 227 (2005), no. 1, 220-226.

[28] Suzuki, T., Takahashi, R., Zhang, X: Extremal boundedness of a variational functional in point vortex mean field theory associated with probability measures, arXiv:1412.4901

(Tonia Ricciardi) Dipartimento di Matematica e Applicazioni, Università di Napoli Federico II, Via Cintia, Monte S. Angelo, 80126 Napoli, Italy

E-mail address: tonricci@unina.it

(Ryo Takahashi) Division of Mathematical Science, Department of Systems Innovation, Graduate School of Engineering Science, Osaka University, Machikaneyama-Cho 1-3, Toyonaka-Shi, 560-8531, JAPAN

E-mail address: r-takaha@sigmath.es.osaka-u.ac.jp

(Gabriella Zecca) Dipartimento di Matematica e Applicazioni, Università di Napoli Federico II, Via Cintia, Monte S. Angelo, 80126 Napoli, Italy

E-mail address: g.zecca@unina.it

(Xiao Zhang) Division of Mathematical Science, Department of Systems Innovation, Graduate School of Engineering Science, Osaka University, Machikaneyama-Cho 1-3, Toyonaka-Shi, 5608531, JAPAN

E-mail address: zhangx@sigmath.es.osaka-u.ac.jp 